\title{
UNION CURVES AND UNION CURVATURE
}

\author{
C. E. SPRINGER
}

Introduction. A rectilinear congruence in ordinary three-dimensional Euclidean space may be defined by specifying the direction of a unique line at each point of a given surface. A union curve ${ }^{1}$ on the surface relative to a given congruence has the property that its osculating plane at each point of the curve contains the line of the congruence through the point. It is well known that the union curves relative to the congruence of normals to a surface are the geodesic curves on the surface. The principal aim of this paper is to generalize for union curves certain known results concerning geodesic curves.

The analytical representation of the congruence in $\$ 1$ is followed in $\$ 2$ by the derivation of the differential equations of the union curves referred to an arbitrary system of coordinates on the surface. From the definition of the union curvature vector in $\$ 3$, it is seen that a union curve on a surface may be defined as a curve for which the union curvature vector is a null vector at every point of the curve. Finally, there appears in $\$ 4$ a geometric interpretation of the union curvature of a curve on a surface which agrees with the definition of geodesic curvature of the curve for the particular case of the congruence of normals to the surface.

The notation of Eisenhart ${ }^{2}$ will be employed for the most part, although $\Gamma_{\beta \gamma}^{\alpha}$ will be used here as the Christoffel symbol of the second kind. Greek indices will always take the range 1, 2, and Latin indices the range $1,2,3$. The summation convention of the tensor analysis will be observed.

1. Analytical representation of the congruence. Let the surface $S$ be defined analytically with reference to an orthogonal cartesian system of coordinates by

$$
x^{i}=x^{i}\left(u^{1}, u^{2}\right) \quad(i=1,2,3),
$$

where the functions $x^{i}$ and their partial derivatives to the second order are understood to be continuous at any point $P$ on $S$. Let the line $l$ of the congruence at $P$ have direction cosines given by

$$
\lambda^{i}=\lambda^{i}\left(u^{1}, u^{2}\right), \quad \quad \quad \lambda^{i} \lambda^{i}=1,
$$

Received by the editors April 19, 1945.

1 Sperry, Properties of a certain projectively defined two-parameter family of curves on a general surface, Amer. J. Math. vol. 40 (1918) p. 213.

2 Eisenhart, Differential geometry, Princeton University Press, 1940. 
where the functions $\lambda^{i}$ are continuous at $P$. If $X^{i}$ denote the direction cosines of the normal to $S$ at $P$, the direction cosines of $l$ at $P$ may be written in the form

$$
\lambda^{i}=p^{\alpha} x_{, \alpha}^{i}+q X^{i} \quad(q>0),
$$

where, for convenience, the notation of the covariant derivative $x_{, \alpha}^{s}$ of $x^{i}$ with respect to the first fundamental tensor $\left(g_{\alpha \beta} \equiv x_{, \alpha}^{*} x_{, \beta}^{*}\right)$ of $S$ is used instead of $\partial x^{i} / \partial u^{\alpha}$. By virtue of the second of equations (2), there results

$$
\lambda^{i} \lambda^{i} \equiv\left(p^{\alpha} x_{, \alpha}^{i}+q X^{i}\right)\left(p^{\beta} x_{, \beta}^{i}+q X^{i}\right) \equiv g_{\alpha \beta} p^{\alpha} p^{\beta}+q^{2}=1 .
$$

If $\theta$ is the angle between $l$ at $P$ and the normal to $S$ at $P$, it is seen from equation (3) that

$$
\cos \theta=\lambda^{i} X^{i}=q,
$$

and from (4) that the magnitude of the vector with contravariant components $p^{\alpha}$ is $\sin \theta$. It may be noticed also that if $C$ is any curve on $S$ through $P$ represented by $u^{\alpha}=u^{\alpha}(s)$, where $s$ denotes arc length, then the cosine of the angle $\phi$ between $l$ and the direction of the tangent to $C$ at $P$ is given by

$$
\cos \phi=\lambda^{i} \frac{d x^{i}}{d s}=\left(p^{\alpha} x_{, \alpha}^{i}+q X^{i}\right) x_{, \beta}^{i} \frac{d u^{\beta}}{d s}=g_{\alpha \beta} p^{\alpha} \frac{d u^{\beta}}{d s} .
$$

2. Differential equations of the union curves. The osculating plane to the curve $C: u^{\alpha}=u^{\alpha}(s)$ on $S$ at $P$ has the determinantal equation

$$
\delta_{i j k}^{123}\left(\bar{x}^{i}-x^{i}\right) \frac{d x^{i}}{d s} \frac{d^{2} x^{k}}{d s^{2}}=0,
$$

the $\bar{x}^{i}$ being current coordinates. On writing

$$
\frac{d x^{j}}{d s}=x_{, \sigma}^{j} u^{\prime \sigma}, \quad \frac{d^{2} x^{k}}{d s^{2}}=x_{, \tau}^{k} u^{\prime \prime \tau}+\frac{\partial^{2} x^{k}}{\partial u^{\alpha} \partial u^{\beta}} u^{\prime{ }^{\alpha}} u^{{ }^{\beta}},
$$

where the primes indicate differentiation with respect to $s$, and, in turn, the Gauss equations of the surface $S$, namely,

$$
\frac{\partial^{2} x^{k}}{\partial u^{\alpha} \partial u^{\beta}}=\Gamma_{\alpha \beta}^{\gamma} x_{, \gamma}^{k}+d_{\alpha \beta} X^{k},
$$

we see that equation (7) takes the form

$$
\delta_{i j k}^{123}\left(x^{i}-x^{i}\right)\left(u^{\prime \sigma} x_{, \sigma}^{j}\right)\left(\rho^{\top} x_{, \tau}^{k}+d_{\alpha \beta} u^{\prime \alpha} u^{\prime \beta} X^{k}\right)=0,
$$


where $\rho^{\tau}$, the components of the curvature vector ${ }^{3}$ of $C$ at $P$, are given by

$$
\rho^{\tau} \equiv u^{\prime \prime \tau}+\Gamma_{\alpha \beta}^{\tau} u^{\prime \alpha} u^{\prime \beta} .
$$

If the osculating plane to $C$ at $P$ contains line $l$, the coordinates $x^{i}+t \lambda^{i} \equiv x^{i}+t\left(p^{\gamma} x_{, \gamma}^{i}+q X^{i}\right)$ must satisfy equation (10) for all $t$. Thus, the condition

$$
\delta_{i j k}^{123}\left(p^{\gamma} x_{, \gamma}^{i}+q X^{i}\right)\left(u^{\prime \sigma} x_{, \sigma}^{j}\right)\left(\rho^{\tau} x_{, \tau}^{k}+d_{\alpha \beta} u^{\prime \alpha} u^{\prime \beta} X^{k}\right)=0
$$

must obtain. Use of the facts that $\delta_{1, k}^{123} x_{, \gamma}^{k} x_{, \sigma}^{j} x_{, \tau}^{k} \equiv 0$ (because $\gamma, \sigma, \tau$ cannot all be different) and $\delta_{l j k}^{123} X^{i} x_{, \sigma}^{j} X^{k} \equiv 0$, together with a change of umbral indices, allows equation (12) to take the form

$$
\left(\delta_{i j k}^{123} X^{i} x_{, \sigma}^{j} x_{, \tau}^{k}\right)\left(p^{\sigma} u^{\prime \tau} d_{\alpha \beta} u^{\prime \alpha} u^{\prime \beta}+q u_{\rho}^{\prime \sigma} \rho^{\tau}\right)=0 .
$$

It is to be noticed here that $d_{\alpha \beta} u^{\prime \alpha} u^{\prime \beta}$ is the normal curvature $K_{n}$ of the curve $C$ with direction $u^{\prime \alpha}(\alpha=1,2)$ on the surface $S$.

Summing on $\sigma$ and $\tau$ in equation (13), and neglecting the nonzero determinant $\delta_{\mathbf{l} j k}^{123} X^{i} x_{, 1}^{j} x_{, 2}^{k}$, we obtain

$$
e_{\sigma \tau}\left(p^{\sigma} u^{\prime \tau} K_{n}+q u^{\prime \sigma} \rho^{\tau}\right)=0,
$$

where $^{4} e_{12}=1, e_{21}=-1, e_{11}=e_{22}=0$.

Equation (14) is the differential equation of second order of the union curves on the surface $S$ through any point $P$ on $S$, the parametric curves being any whatever. Lan ${ }^{5}$ has given the differential equation of union curves on a metric surface for the case in which the lines of curvature on the surface are taken as parametric.

From equation (14) one may conclude that if $q=0$ (which means that $l$ lies in the tangent plane to $S$ at $P$ ), then the only union curves are those in the directions given by $p^{1} d u^{2}-p^{2} d u^{1}=0$ and by $K_{n}$ $\equiv d_{\alpha \beta} u^{\prime \alpha} u^{\prime \beta}=0$, the asymptotic directions. Suppose, henceforth, that $q \neq 0$, and let $l^{\sigma}$ be written for $p^{\sigma} / q$. Then, if $\sigma$ and $\tau$ be interchanged in the second term of equation (14), the differential equation of the union curves on $S$ through $P$ becomes

$$
e_{\sigma \tau} u^{\prime \sigma}\left(\rho^{\tau}-K_{n} l \tau\right)=0 .
$$

If the components $\rho^{\tau}$ of the curvature vector of the curve are zero, the curve is a geodesic. From equation (15), it can be seen that the

${ }^{3}$ Eisenhart, loc. cit. p. 187.

${ }^{4}$ Eisenhart, loc. cit. p. 135.

${ }^{-}$Lane, Projective differential geometry of curves and surfaces, University of Chicago Press, 1931, p. 240. 
geodesics and union curves on a surface coincide in the three directions given by $p^{2} d u^{1}-p^{1} d u^{2}=0$ and the directions of the asymptotic curves on the surface. Moreover, it may be seen from equation (15) that if the congruence is normal to the surface $\left(l^{\sigma}=0\right)$, the union curves are geodesic curves on $S$.

3. Union curvature of a curve on a surface. Equation (15) is a single differential equation of the second order. It will be shown to be equivalent to a pair of differential equations of the second order.

The curvature vector with components $\rho^{\tau}$ is orthogonal to the direction $u^{\prime \alpha}$ of $C$ on $S$. Hence ${ }^{6}$

$$
g_{\alpha \beta} \rho^{\alpha} u^{\prime \beta}=0 .
$$

If equation (16), multiplied by $u^{\prime 1}$, and equation (15), multiplied by $g_{2 \beta} u^{\prime \beta}$, are subtracted, there results, by use of $g_{\alpha \beta} u^{\prime \alpha} u^{\prime \beta}=1$, the first of the following differential equations of the union curves on $S$,

$$
\begin{aligned}
& \eta^{1} \equiv \rho^{1}-K_{n} g_{2 \beta} u^{\prime \beta} \cdot e_{\sigma \tau} l^{\sigma} u^{\prime \tau}=0, \\
& \eta^{2} \equiv \rho^{2}+K_{n} g_{1 \beta} u^{\prime \beta} \cdot e_{\sigma r} l^{\sigma} u^{\prime \tau}=0,
\end{aligned}
$$

and the second equation is obtained in a similar manner. The vector with components $\eta^{\alpha}$ given by (17) lies in the tangent plane to $S$ at $P$. It may be called the union curvature vector of the curve $C$ on $S$ at $P$. It may be concluded from equations (17) that the union curvature vector is a zero vector at each point of a union curve.

The geodesic curvature ${ }^{7} K_{v}$ of the curve $C$ at $P$ is given by

$$
K_{o} \equiv \epsilon_{\alpha \beta} u^{\prime \alpha} \rho^{\beta},
$$

where $\epsilon_{\alpha \beta}=g^{1 / 2} e_{\alpha \beta}$. Therefore, it appears appropriate to define the union curvature $K_{u}$ of $C$ at $P$ by

$$
K_{u} \equiv \epsilon_{\alpha \beta} u^{\prime \alpha} \eta^{\beta},
$$

which may be written, by use of equations (16) and (17), in the form

$$
K_{u} \equiv \epsilon_{\sigma \tau} u^{\prime \sigma}\left(\rho^{\tau}-K_{n} l^{\tau}\right)=K_{\sigma}-K_{n} \epsilon_{\sigma \tau} u^{\prime \sigma} l^{r} .
$$

It may be observed from equation (20) that the geodesic curvature along a union curve $\left(K_{u} \equiv 0\right)$ is given by

$$
K_{\theta}=K_{n} \epsilon_{\sigma \tau} u^{\prime \sigma l}{ }^{r} \text {. }
$$

From equation (15) it is seen that the coordinate curves on the surface are union curves if, and only if, the congruence is specified by

- Eisenhart, loc. cit. p. 171.

'Eisenhart, loc. cit. p. 187. 


$$
l^{1}=\Gamma_{22}^{1} / d_{22}, \quad l^{2}=\Gamma_{11}^{2} / d_{11} .
$$

For this particular congruence, the geodesic curvature of any union curve on $S$ at $P$ is given by

$$
K_{v}=g^{1 / 2}\left(\frac{\Gamma_{11}^{2}}{d_{11}} u^{\prime 1}-\frac{\Gamma_{22}^{1}}{d_{22}} u^{\prime 2}\right) d_{\alpha \beta} u^{\prime \alpha} u^{\prime \beta}
$$

If $K_{v_{1}}, K_{v_{2}}$ denote the geodesic curvatures of the curves through $P$ represented by $d u^{2}=0, d u^{1}=0$, it can be seen from equation (23) that the sum of the geodesic curvatures of the coordinate curves on a surface is given by

$$
K_{\sigma_{1}}+K_{\theta_{2}}=g^{1 / 2}\left[\Gamma_{11}^{2}\left(u^{\prime 1}\right)^{3}-\Gamma_{22}^{1}\left(u^{\prime 2}\right)^{3}\right] .
$$

When the asymptotic curves are taken as parametric, the directions of Segre ${ }^{8}$ at the point $P$ on the surface are given by $\Gamma_{11}^{2}\left(u^{\prime 1}\right)^{3}-\Gamma_{22}^{1}\left(u^{\prime 2}\right)^{3}$ $=0$. Hence, it can be concluded that the directions of Segre are those in which the geodesic curvatures of the asymptotic curves differ in sign. This may be compared with the result ${ }^{9}$ that the torsions of the asymptotic curves through any point differ in sign.

4. Geometric interpretation of union curvature. The geodesic curvature of a curve $C$ at $P$ on the surface is the curvature of the curve obtained by projecting the curve $C$ orthogonally onto the tangent plane to the surface at $P$. Let the curve $C$ be projected onto the tangent plane to the surface at $P$ in the direction of the line $l$ of the congruence. It will be shown that the curvature of the plane curve $C^{\prime}$ thus obtained is given by the expression in equation (20).

Let the cylindrical surface of projection of curve $C$ onto the tangent plane at $P$ in the direction of line $l$ at $P$ be denoted by $S^{\prime}$. If $1 / R$ is the normal curvature of $C$ as a curve on $S^{\prime}$ at $P$, and $\alpha$ is the angle between the principal normal to $C$ at $P$ and the normal to the plane of $l$ and the tangent line to $C$ at $P$, then, by the theorem of Meusnier,

$$
e / R=\rho^{-1} \cos \alpha,
$$

where $e= \pm 1$, and $1 / \rho$ is the curvature of $C$ at $P$. Further, if $1 / r$ is the curvature of the curve $C^{\prime}$ at $P$, and if $\beta$ is the angle between the tangent plane to $S$ at $P$ and the plane through the tangent line to $C^{\prime}$ normal to the plane of $l$ and this tangent line, then, again by the theorem of Meusnier,

\footnotetext{
${ }^{8}$ Lane, loc. cit. p. 77; also Springer, Bull. Amer. Math. Soc. vol. 48 (1942) pp. 901906.

${ }^{9}$ Eisenhart, loc. cit. p. 248.
} 


$$
e / R=r^{-1} \cos \beta .
$$

Therefore, by equations (25) and (26)

$$
e / r=\cos \alpha /(\rho \cos \beta) \text {. }
$$

Substitution of the analytical expressions for $\cos \alpha$ and $\cos \beta$ into equation (27) yields, after some simplification,

$$
e / r=\epsilon_{\sigma \tau} u^{\prime \sigma}\left(\rho^{\tau}-K_{n} l \tau\right),
$$

which is the union curvature $K_{u}$ given in equation (20). Therefore, the union curvature of a curve $C$ at a point $P$ on a surface $S$ relative to a given congruence is the curvature of the curve obtained by projecting $C$ onto the tangent plane to $S$ at $P$ in the direction of the line $l$ of the congruence at $P$.

UNIVERSITY OF OKLAHOMA 